\title{
Erratum to: Quality of Recovery After Low-Pressure Laparoscopic Donor Nephrectomy Facilitated by Deep Neuromuscular Blockade: A Randomized Controlled Study
}

Denise M. D. Özdemir-van Brunschot ${ }^{1} \cdot$ Gert J. Scheffer $^{2} \cdot$ Michel van der Jagt ${ }^{1}$ Johan F. Langenhuijsen ${ }^{3}$ - Albert Dahan ${ }^{4} \cdot$ Janneke E. E. A. Mulder $^{2}$ • Simone Willems ${ }^{2} \cdot$ Luuk B. Hilbrands $^{5} \cdot$ Rogier Donders $^{6} \cdot$ Cees J. H. M. van Laarhoven $^{1}$ • Frank A. d'Ancona ${ }^{3} \cdot$ Michiel C. Warlé ${ }^{1}$

Published online: 18 August 2017

(c) Société Internationale de Chirurgie 2017

\section{Erratum to: World J Surg}

DOI 10.1007/s00268-017-4080-x

In the original article Johan F. Langenhuijsen's given name was incorrect. It is correct as reflected in this erratum.

The online version of the original article can be found under doi:10. 1007/s00268-017-4080-x.

Michiel C. Warlé

Michiel.Warle@radboudumc.nl

1 Department of Surgery, Radboud University Medical Center, Geert Grooteplein-Zuid 10, 6525 GA Nijmegen,

The Netherlands

2 Department of Anesthesiology, Radboudumc, Nijmegen, The Netherlands

3 Department of Urology, Radboudumc, Nijmegen, The Netherlands

4 Department of Anesthesiology, Leiden University Medical Centre, Leiden, The Netherlands

5 Department of Nephrology, Radboudumc, Nijmegen, The Netherlands

6 Department of HTA, Radboudumc, Nijmegen, The Netherlands 